\title{
diffusion-fundamentals.org
}

The Open-Access Journal for the Basic Principles of Diffusion Theory, Experiment and Application

\section{Assessment of GABARAP self-association by its diffusion properties}

\author{
Víctor Hugo Pacheco Torres \\ Institute of Complex Systems (ICS-6), Forschungszentrum Jülich, Jülich, Germany \\ vpachecotorres@googlemail.com
}

\begin{abstract}
Gamma-aminobutyric acid type A-receptor-associated protein (GABARAP) belongs to a family of small ubiquitin-like adaptor proteins implicated in intracellular vesicle trafficking and autophagy. Biochemical evidence, as well as observations from X-ray crystallography, supports the view that GABARAP shows a propensity to oligomerize in solution. Here, we have used diffusion-ordered nuclear magnetic resonance spectroscopy (DOSY-NMR) to study the temperature and concentration dependence of the diffusion properties of GABARAP. Our data suggest the presence of distinct conformational states and provide support for self-association of GABARAP molecules. Assuming a monomerdimer equilibrium, the mass fraction of the dimer could be determined for every condition. Based on a series of $1 \mathrm{H}-15 \mathrm{~N}$-heteronuclear single quantum coherence (HSQC) NMR spectra recorded at different temperatures, we propose residues potentially involved in GABARAP self-interaction.
\end{abstract}

